\title{
Locked nailing for the treatment of displaced articular fractures of the calcaneus: description of a new procedure with Calcanail. Answer to Xin Wang, Jia-qian Zhou, Guang-rong Yu
}

\author{
Patrick Simon
}

Received: 28 May 2012/Accepted: 28 May 2012/Published online: 7 September 2012

(C) Springer-Verlag 2012

\section{Dear Colleagues}

Firstly, we would deeply thank you for your kind comments about "our hard works and innovative design on calcaneal fractures research".

Thank you also for your very relevant questions.

What is the indication of the procedure? As you noted yourself, it's easier to reduce by inside an entire depressed articular surface. However, an intra-articular fracture with a vertically depressed lateral fragment and a horizontally medial depressed fragment may be managed with our technique, and this is not so difficult. Of course more complex fractures with more than one intra-articular separation fracture line should be better treated by a primary arthrodesis.

The evaluation of a residual intra-articular step? It's not so easy to evaluate such a residual step perioperatively. We don't perform arthroscopic control. The stability of the construct could probably explain good functional results even in case of a residual articular step.

The iatrogenic damage of the articular surface? We never damaged the articular surface during reaming. Of course take care not to push so far the reamer is crucial!

How to manage the reduction of the calcaneocuboid joint? This is a very good question. Our answer is that in our experience, malreduction of this joint is rarely symptomatic and post-traumatic arthritis very rare. So we don't reduce, specifically, this calcaneocuboid joint in our technique.

How to correct the expansion malformation? Two points contribute to this correction: firstly, the distraction device allows a good restoration of the height of the calcaneus, which greatly reduces the expansion deformity. Secondly, our screws with large conical heads provide a compressive effect.

The impingement of peroneal tendons? We never saw any problem related to peroneal tendons with our procedure, and this is a major progress in relation to older techniques.

The removal of the nail? Removal of the nail after consolidation was never performed until now. This has to be done by using the arthrodesis hollow reamer which is $12 \mathrm{~mm}$ in diameter: this will cut bony union through the windows of the nails.

We hope our answers will convince you of the safety of our device and thank you again for your letter.

Conflict of interest No funds were received in support of this study. 